\title{
Options for reststrahlen materials in optical surfaces and filters
}

\author{
S. Zhao* and C-G. Ribbing \\ Division of Solid State Physics, Angström Laboratory, Uppsala University, \\ Box 534, SE-751 21 Uppsala, Sweden \\ *E-mail: shuxi.zhao@angstrom.uu.se \\ Received October 31, 2009
}

\begin{abstract}
The current and potential applications of reststrahlen bands in optical surfaces and components are reviewed. This type of interval with metallic-like high reflectance has been used for monochromatization in infrared and when wavelength-selective low emittance is needed for frost prevention or infrared signature reduction. Associated features, including a narrow reflectance minimum for a wide range of angles of incidence, are potentially useful.
\end{abstract}

OCIS codes: $160.3220,1604670,160.6030,350.2770$.

doi: 10.3788/COL201008Sl.0119.

A reststrahlen material is a compound with at least partly ionic bonding. This entails that a net of alternating positively and negatively charged ion cores are arranged in a periodic pattern that constitutes the lattice of the compound. This lattice can be excited by transverse electromagnetic (EM) waves that accelerate the positive and negative ions in opposite directions.

The excitation can be modeled as a damped harmonic oscillator. The oscillator has a resonance that corresponds to energy absorption. Simple considerations of ionic bonding of atomic masses in real compounds show that this resonance occurs in the infrared (IR) frequency range. In analogy with a mechanical oscillator, the resonance frequency increases with the bonding strength and decreases with the effective molecular mass ${ }^{[1]}$.

An IR wave traveling through a lattice has a wavelength that is much longer than the lattice constant and the typical distances between neighboring ions. The location of this excitation in the phonon spectrum of the compound is usually associated with the transverse optical (TO) branch. Considering the long wavelength, this should be close to the origin in $k$-space (i.e., $k \approx 0$ ). The frequency of the TO-branch intersecting with the frequency axis is conventionally labeled as $\omega_{\mathrm{T}}{ }^{[2]}$. We can thus associate this molecular oscillator resonance frequency with a particular feature in the phonon spectrum of the crystal. The combined excitation of EM wave with phonon traveling through the crystal is named polariton. Using the analogy of the mechanical oscillator, which is accelerated by the electric vector of an EM wave, the classical Lorentz model dielectric function is derived ${ }^{[2]}$ by

$$
\tilde{\varepsilon}(\omega)=\varepsilon_{\infty}+\frac{N e^{2}}{\varepsilon_{0} \mu} \frac{1}{\left(\omega_{T}^{2}-\omega^{2}\right)-i \omega \Gamma},
$$

where the screening constant $\varepsilon_{\infty}$ approximates the sum of all contributions from high-frequency excitations, and $N$ and $\mu$ are the volume density and reduced mass, respectively, of the molecules that form the crystal. $\Gamma$ is a phenomenological "friction" with dimension of angular frequency representing collisions (i.e., losses for the polaritons travelling through the lattice). It is characteristic that an imaginary part of the complex dielectric function be obtained via the losses. Figure 1 shows the real and imaginary parts of the dielectric function together with the bulk normal reflectance on a normalized frequency axis.

The prominent peak in the $\varepsilon_{2}$-function at frequency $\omega_{\mathrm{T}}$ represents the losses, and the width of this peak is proportional to $\Gamma$. Also notable is the interval where the $\varepsilon_{1}$-function is $<0$. In this case, it is $1<\omega / \omega_{\mathrm{T}}<1.65$, but the value of the upper limit is dependent upon the choice of parameters. Introducing $\omega_{\mathrm{p}}$, the local oscillator (LO)-frequency for $k=0$ is

$$
\omega_{\mathrm{p}}=\sqrt{\frac{N e^{2}}{\varepsilon_{0} \mu}} .
$$

This is the upper zero in the absence of screening (i.e., $\varepsilon_{\infty}=1$ ). For weak damping (i.e., $\Gamma<<\omega_{0}$ ), we obtain the upper limit, $\omega_{\mathrm{p}}^{\prime}$, of the reststrahlen band as

$$
\frac{\omega_{\mathrm{p}}^{\prime}}{\omega_{\mathrm{T}}} \approx \sqrt{1+\frac{1}{\varepsilon_{\infty}}\left(\frac{\omega_{\mathrm{p}}}{\omega_{\mathrm{T}}}\right)^{2}} .
$$

The screening constant corresponds to a rigid shift upwards of the $\varepsilon_{1}(\omega)$-curve. Thus, the width of the negative $\varepsilon_{1}(\omega)$-interval is reduced by screening.

The negative $\varepsilon_{1}$-values give high bulk reflectance, as seen in the diagram. In the case that there is a high bulk reflectance interval just above an oscillator resonance, the interval is called a reststrahlen band. The textbook cases of these bands are ionic crystals with dominant ionic bonding and high-reflectance bands typically in the wavelength range of $50-200 \mu \mathrm{m}$. It is notable that the high reflectance values of reststrahlen bands are obtained without the presence of mobile conduction electrons; the ionic compounds are electrical insulators.

The appearance of reststrahlen bands goes well beyond the group of alkali halides and others with close to $100 \%$ ionic bonding. An extensive documentation of these bands ${ }^{[3]}$ contains several light and hard compounds 


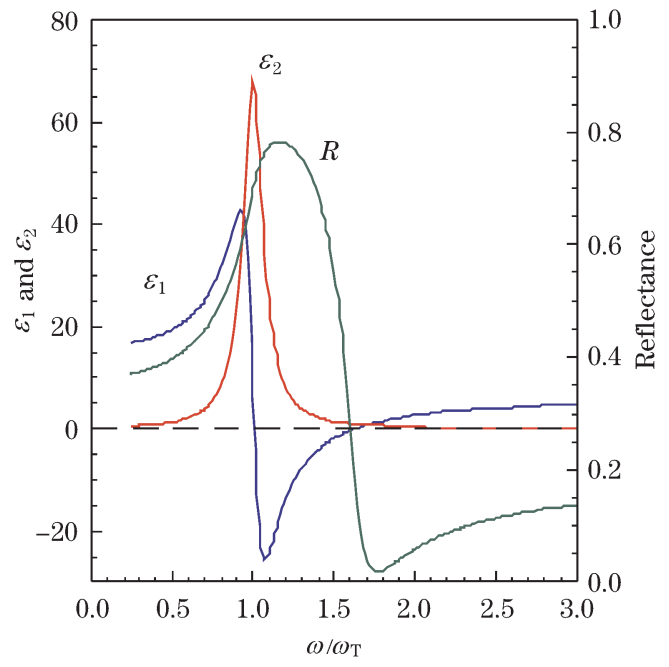

Fig. 1. The Lorentz single oscillator model dielectric function and the corresponding bulk, normal reflectance. Parameter values: $\varepsilon_{\infty}=6, \Gamma / \omega_{0}=0.15$, and $\omega_{\mathrm{p}} / \omega_{\mathrm{T}}=3.19$.

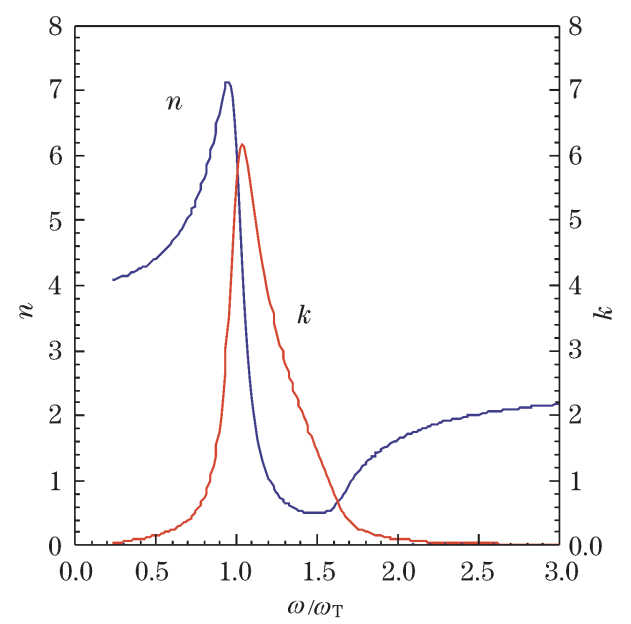

Fig. 2. The Lorentz single oscillator model optical functions $n(\lambda)$ and $k(\lambda)$ vs. thenormalized frequency $\omega / \omega_{\mathrm{T}}$ (with the same parameter values as in Fig. 1).

such as $\mathrm{BN}, \mathrm{BeO}$, and $\mathrm{Al}_{2} \mathrm{O}_{3}$ with mixed ionic and covalent bonding of light atoms, which have prominent Reststrahlen bands at shorter wavelengths of $5-20 \mu \mathrm{m}$. This gives us electrically insulating compounds that feature high reflectance in the thermal infrared part of the spectrum, which is the core object of this review.

In order to identify more important features, we plot in Fig. 2 reflactive index $n(\lambda)$ and extinction coefficient $k(\lambda)$ for the same single oscillator model as in Fig. 1. In Fig. 2, the sharp peaks of $n$ and $k$ are noticeable on either side of the absorption frequency $\omega_{\mathrm{T}}$, as well as in the characteristically low $n$-values of $<1$ in the reststrahlen region. This interval is thus characterized by $k>>n$, which again shows that the insulating compound in the reststrahlen region has metal-like optical behavior. We observe that $n=1$ has two solutions: one occurs for $k>1$, while the other for $k<<1$. If there are no other resonances between IRs and the visible, the high-energy asymptote $n_{\infty}=\sqrt{\varepsilon_{\infty}}$ is an approximate value for $n_{\mathrm{VIS}}$.

In this letter, various examples of established and suggested uses of reststrahlen compounds in optical components are described. We start with the most obvious end, using the reststrahlen band as a reflector and then move to applications based on other typical features. These include the two intersections of the $n$-curve and $k$-curve in Fig. 2, which result in $\varepsilon_{1}=0$, as well as one of the two solutions for $n=1$. The interval with $\varepsilon_{1}<0$ can also be used for effects other than high reflectance since it is a condition for the generation of interface plasmonpolaritons. Again, the metal-like optical behavior in this IR-band allows for these potential applications.

The classical application, which is also an explanation for the German denomination reststrahlen, uses multiple reflections in a chosen compound for monochromatization of infrared light. In the past, this technique was used for IR spectrocopy ${ }^{[4]}$. The shallow edge of the reflectance on the long wavelength side of the reststrahlen band limits the narrowness of the spectral width of the light obtained with this method. Turner et al. demonstrated that a thin dielectric layer on the compound improves performance ${ }^{[5]}$. The result for $\mathrm{MgO}$ coated with $\mathrm{PbCl}_{2}$ is shown in Fig. 3.

The thin film coating not only sharpens the long wavelength edge of the band, but also raises the peak reflectance. Both features help improve performance. However, with the development of gratings for spectrophotometers, this application has rapidly become outdated. Thus, this study returns to the use of a dielectric coating on a reststrahlen compound to modify the optical behavior. However, before delving into this, there is a need to describe omnidirectional antireflection, a seemingly opposite application that has been suggested and studied in considerable detail by Dobrowolski et al. ${ }^{[6,7]}$. This application is based upon a typical oscillator feature, although just outside the proper Restrahlen band. As seen in Figs. 1 and 3, it is a characteristic of a screened oscillator model that the bulk reflectance has a minimum on the high frequency side of the band. The $n$-curve in Fig. 2 has two solutions when $n=1$. The high frequency solution typically exhibits $k<<1$, which results in very low bulk reflectance. In Fig. 4, we cite the calculated average reflectance spectra for s- and p-polarized light at various angles of incidence. The optical data used are published values for $\mathrm{SiO}_{2}$-glass, which exhibits this behavior for $\lambda=7.2 \mu \mathrm{m}$. The minimum remains even with increasing the angle of incidence, although it is narrowed by the widening of the reststrahlen band. The calculations demonstrate that the reflectance at $\lambda$ $=7.2 \mu \mathrm{m}<0.05$ for all angles of incidence $<85^{\circ}$. This is remarkable, but the usefulness is limited by the supply of reststrahlen materials that determine at what wavelengths to which this can be realized and the nar-

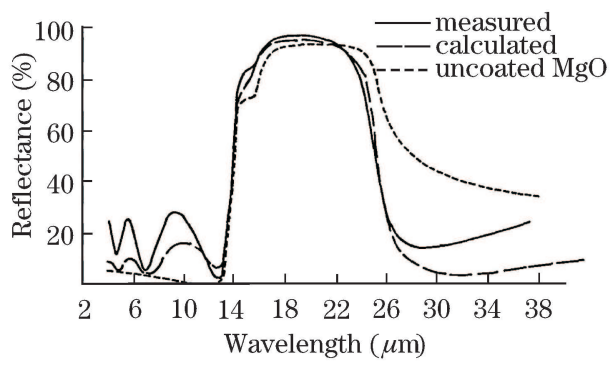

Fig. 3. Measured and calculated near normal reflectance for $\mathrm{PbCl}_{2}$ on $\mathrm{MgO}$ compared with the measured reflectance for $\mathrm{MgO}$ alone ${ }^{[5]}$. 


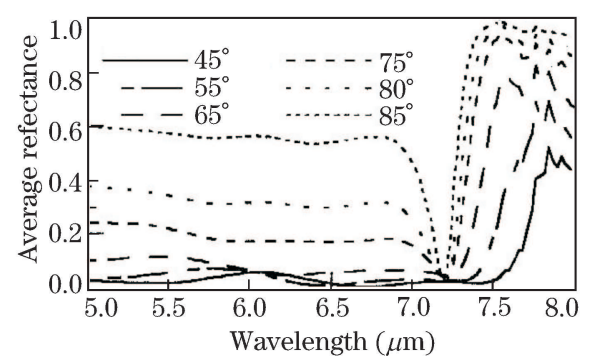

Fig. 4. $\mathrm{SiO}_{2}$ averaged s- and p-polarized reflectance for various angles of incidence on a four-layer coating with $\mathrm{SiO}_{2}$-glass on top, anti-reflecting a substrate with $n=3^{[6]}$.

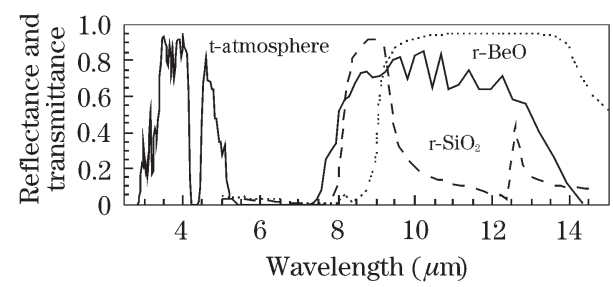

Fig. 5. The mid-latitude atmospheric transmittance spectrum compared with the reflectance spectra featuring the Reststrahlen bands of $\mathrm{SiO}_{2}$ and $\mathrm{BeO}$.

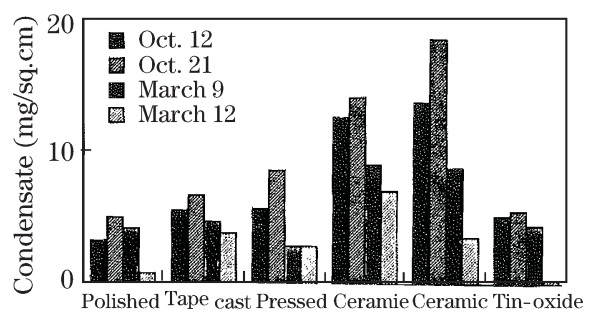

Fig. 6. Measured amount of frost/unit area grown on different samples as indicated. The three left-most categories are the different qualities of BeO. "Ceramics" are typical glazed silicate insulator materials. "Tin-oxide" is ordinary glass coated with conducting, doped pyrolytic $\mathrm{SnO}_{2}$ (cited from Ref. [8]).

row interval in which this angular insensitivity is present.

Kirchhoff's law of radiation applied to an opaque body results in the dimensionless intensity ratios for intensity reflectance $(R)$ and fractional emissivity $(\varepsilon)$. The simple relation is

$$
\varepsilon(\lambda)=1-R(\lambda) .
$$

It shows that a restrahlen band can be used to create a wavelength band with low emittance.

The preferential growth of frost on an outdoor surface is often caused by radiative cooling, particularly on dry, calm nights. If a surface has high emittance in the upper atmospheric window wavelength range of 8-13 $\mu \mathrm{m}$, objects at normal outdoor temperatures will have strong radiative exchange with the cold tropopause or even the outer space. This explains why objects can become colder than the surrounding air and why water in the desert may freeze even if the air temperature is at $>0{ }^{\circ} \mathrm{C}$. Conversely, if a material has a reststrahlen band that reduces thermal emission in this window, frost deposition may be avoided. In Fig. 5, two examples are compared with the transmittance spectrum of a dry atmosphere. The crucial parameter is the extent of the Reststrahlen band which "overlaps" the atmospheric transmittance, thereby blocking the emission to upper layers in the atmosphere.
It has been demonstrated that less frost grows on ceramic beryllium oxide, the reststrahlen band of which covers a wavelength of $9.1-14.9 \mu \mathrm{m}$, than on other related materials ${ }^{[8,9]}$. Frost deposition on high-power china insulators, which may cause short-circuiting during thawing. The excellent electrical insulating properties of $\mathrm{BeO}$ are therefore essential. In Fig. 6, we cite the result of this case study. The amount of frost grown during four different clear nights is compared for three different $\mathrm{BeO}$ samples (i.e., two ordinary ceramic insulators and one sample of tin-oxide-coated glass), which are suggested for frost prevention on car windows ${ }^{[10,11]}$. It should be noted that this last sample has high surface conductivity and could therefore not be used on electric insulators.

In a dynamic description, the heat content and conductivity of the bulk insulator material should also be considered. Ordinary glass has generally high emittance modulated by a weak reststrahlen band that does not overlap the atmospheric window. Furthermore, it is a poor heat conductor. This is also true for most polycrystalline silicates. As an example, we consider a car parked outdoor (as in Refs. [10] and [11]). We assume a clear night when cooling by convection, conduction, and radiation. If the latter dominates, it is understandable that the glass is preferentially frost-coated in comparison with the metallic surfaces, which are non-selectively lowemitting with high thermal conductance.

For the insulator application, the electric behavior is of primary concern. A significantly lower amount of frost grows on the $\mathrm{BeO}$ surface than on the ordinary ceramic insulator. The issue on whether long-term exposure to atmospheric pollution increases the low emittance value is thus important. The emittance spectra for $\mathrm{BeO}$ ceramic samples at $100{ }^{\circ} \mathrm{C}$, subjected to standardized contamination with salt and hydrocarbons, were therefore measured $^{[12]}$.

The use of $\mathrm{BeO}$ in industrial processing is limited by the health hazards connected with inhaling $\mathrm{BeO}$ powder. Nevertheless, the $\mathrm{BeO}$ ceramic substrates used in the above laboratory studies are being used in technical applications because they possess an unusual combination of electrical insulation and high thermal conductivity.

The low emissivity of $\mathrm{BeO}$ in the upper atmospheric window has received attention for signature reduction applications. Thermal seekers for moderately hot objects are adapted to work in the upper thermal window of 8$13 \mu \mathrm{m}$. The relevant parameter for the signature in this case is the window emittance

$$
\bar{\varepsilon}_{a b}(T)=\frac{\int_{a}^{b} \varepsilon(\nu) L_{b b}(\nu, T) \mathrm{d} \nu}{\int_{a}^{b} L_{b b}(\nu, T) \mathrm{d} \nu},
$$

where $a$ and $b$ are frequency or energy units for the upper atmospheric window.

Figures 4 and 5 indicate that bulk $\mathrm{BeO}$, or an object coated with $\mathrm{BeO}$, would emit much less than a blackbody in this spectral interval. A detailed investigation gave an emissivity of $\varepsilon_{8-13 \mu \mathrm{m}}\left(50^{\circ} \mathrm{C}\right) \approx 0.27$ for tapecast $\mathrm{BeO}^{[13]}$. Clearly, the basic property of $\mathrm{BeO}-$ low emittance in the upper atmospheric window - makes it promising for both frost prevention and signature reduction. With an object that is hotter than the surrounding 
atmosphere, selectively low emittance offers the added advantage of radiative cooling outside the window not being reduced. A shiny metallic surface would have even lower emittance, but the visual properties would be unacceptable for a military platform, and some radiative cooling would be lost. It should also be noted that painted metal would have $\varepsilon_{(8-13 \mu \mathrm{m})}>0.8$ in most cases.

The question is whether the $\varepsilon_{(8-13 \mu \mathrm{m})}$-value for the $\mathrm{BeO}$ surface could be further improved if the high emission at wavelengths $8-9.5 \mu \mathrm{m}$ is reduced. A high index dielectric film on the $\mathrm{BeO}$ modifies the reflectance spectrum. In particular, a shoulder on the short wavelength edge of the Reststrahlen band can be added, further reducing the emittance in the window interval. This possibility was tested by calculations for a $\mathrm{Si}$ film on an opaque $\mathrm{BeO}$ substrate ${ }^{[13]}$. The resulting integrated emissivity factor for the window 7.7-13 $\mu \mathrm{m}$ as a function of the thickness of the Si film is cited in Fig. 7.

The oscillatory behavior in Fig. 7 may appear as an interference pattern. However, one material is strongly absorbing, so it is not the customary $\lambda / 4$-behavior seen in dielectric multi-layers. The calculated specular minimum value of $\varepsilon_{7.7-13 \mu \mathrm{m}}\left(50{ }^{\circ} \mathrm{C}\right)$ was 0.11 for $d=0.83$ $\mu \mathrm{m}$, as seen in Fig. 7. The corresponding experimental value with a $\mathrm{Si}$ film sputtered onto a $\mathrm{BeO}$ substrate ${ }^{[13]}$ was 0.29 , which is recorded by a filtered radiometer and spectrally with an integrating sphere ${ }^{[13]}$.

As seen in Fig. 5, the band of $\mathrm{SiO}_{2}$ reduces the emittance in the $8-10 \mu \mathrm{m}$ interval, although it is not nearly

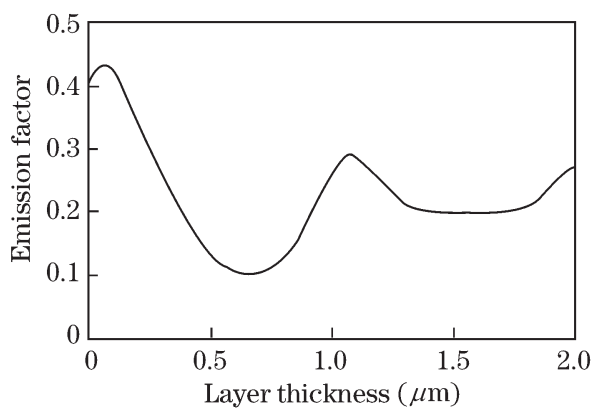

Fig. 7. Calculated specular window emissivity $\varepsilon_{7.7-13 \mu \mathrm{m}}(50$ ${ }^{\circ} \mathrm{C}$ ) for $\mathrm{Si}(\mathrm{d}) / \mathrm{BeO}$ as a function of film thickness (cited from Ref. [13]).

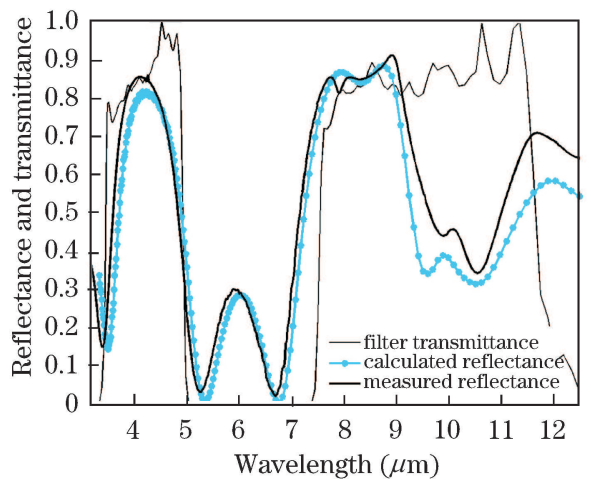

Fig. 8. Calculated and measured reflectance (normal incidence) for the $\mathrm{Si} / \mathrm{SiO}_{2}$ double layer on $\mathrm{Si}$-wafer. The thickness of the top $\mathrm{Si}$ layer is $0.9 \mu \mathrm{m}$, while the $\mathrm{SiO}_{2}$ layer is $2.45 \mu \mathrm{m}$. The figure also contains the transmittance of the filter used in a heat camera sampling the atmospheric windows (cited from Ref. [14]).

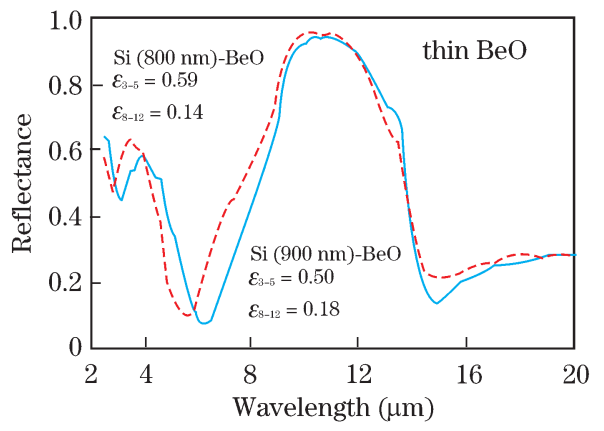

Fig. 9. Measured total reflectance spectra for $\mathrm{Si} / \mathrm{BeO}(\mathrm{Si}$ thickness indicated). The corresponding window-emittance values are also indicated.

as wide and effective as that of BeO. More recently, attempts were made to widen the reststrahlen band of $\mathrm{SiO}_{2}$ with $\mathrm{Si}$ film on top. Double-layer calculations confirmed that this should be achievable. The experimental verification was made with $\mathrm{Si} / \mathrm{SiO}_{2}$ double-layer chemical vaper deposition (CVD) deposited upon a 550$\mu \mathrm{m}$ Si-wafer. This high-temperature process allows for a precise control and results in films with less defects than sputtered. The calculations were made with published optical constants, and the thickness of the film was calibrated from the deposition times. The good agreement between the calculated and the measured curves was thus obtained without fitting. The thin curve in Fig. 8 is the filter curve of a heat camera used to evaluate the signature reduction in integrated emission measurements. Compared to Fig. 5, the Si film has added width to the reststrahlen band of $\mathrm{SiO}_{2}$, which will reduce the signa-

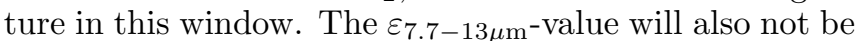
as low as that of $\mathrm{BeO}$. The advantages are practical: the use of non-toxic materials and the availability of a wellcontrolled and well-known deposition process. In addition, this double layer has a second reflectance peak over the lower atmospheric window of $3-5 \mu \mathrm{m}$. The reflectance values are not quite as high, but they coincide well with the lower window, as indicated by the filter curve. This peak is not a direct consequence of the reststrahlen band, but is a characteristic feature: a non-dispersive, dielectric interval at shorter wavelengths than the lattice resonance, as seen in Figs. 1 and 2. This second reflectance peak is thus a normal interference maximum from the combination of high- and low-index dielectric films.

The thickness of the silicone film that positions this interference maximum with $\mathrm{SiO}_{2}$ in the lower atmospheric window also optimizes the shoulders on the $\mathrm{SiO}_{2}$ reststrahlen band ${ }^{[15]}$.

It is technically interesting to reduce the thermal emittance in the lower atmospheric window for at least two reasons. The $8-13 \mu \mathrm{m}$ window contains the blackbody function maximum for moderately high temperatures of $50{ }^{\circ} \mathrm{C}$. However, for hot spots such as exhaust pipes, the $3-5 \mu \mathrm{m}$ interval is more important. Furthermore, the detector for these wavelengths has been improved, thereby increasing their use in IR seekers.

The possibility to reduce the thermal emission in both windows with a single thin film arrangement is attractive, thus the previous $\mathrm{Si}-\mathrm{BeO}$ combination is utilized to determine if the system is equally favorable. The spectrum for bare $\mathrm{BeO}$ (Fig. 5) has an extremely low 
reflectance in the $3-5 \mu \mathrm{m}$ interval. The $\varepsilon_{3-5 \mu \mathrm{m}}$-value is about 0.76 for such a ceramic material. However, the total reflectance of these substrates starts to rise at below $4 \mu \mathrm{m}$ because of the scattering from subsurface inhomogeneities ${ }^{[16]}$. Double-layer calculations such as those used for Fig. 7 were extended to shorter wavelengths, and the parameter $\varepsilon_{3-5 \mu \mathrm{m}}$ was evaluated. The results were sufficiently encouraging to make a new experiment with sputtering Si films on a BeO substrate. The crucial question is whether such a film would increase the reflectance at both wavelengths of 8 and 5 $\mu \mathrm{m}$. In Fig. 9, we show the experimental results for two Si thicknesses: 800 and $900 \mathrm{~nm}$ on a $0.5-\mathrm{mm} \mathrm{BeO}$ substrate.

Both window-emittance parameters have been dramatically improved compared to $\mathrm{BeO}$ alone, even if the calculated minimum cited in Fig. 7 has not been reached and the possible contribution from transmittance was not considered. The results indicated in the diagram also illustrate that the choice of Si film thickness depends on which window should be given priority.

It is notable to mention an invention for the opposite purpose (i.e., to quench a reststrahlen band to increase the thermal emission from a surface). It concerns the glass surface of a solar cell in space, which receives useful visible radiation and solar heat which increases the temperature of the cell and also reduces the efficiency of the cell. In the absence of atmosphere, the cell only cools radiatively. Baumeister and Krisl ${ }^{[17]}$ realized that the reststrahlen band of the glass prevents this radiative cooling to a certain extent. They therefore designed and prepared a $\mathrm{SiO}_{2} / \mathrm{MgF}$ coating that suppresses the reflectance (i.e., increases the emission) in the Reststrahlen region, or an anti-reflection (AR) coating that improves the radiative cooling of a solar cell in space.

In 1998, a report on extraordinary transmission (EOT) received considerable attention ${ }^{[18]}$. Ebbesen et al. reported high transmission for a thin, but opaque, silver film with a square pattern of sub-wavelength circular holes. The transmission was unexpected in three respects. Firstly, it was higher than what was obtained from multiplying the number of holes utilizing the wellknown Bethe-value ${ }^{[19]}$ for a single hole. Secondly, the transmitted intensity was higher than the total intensity impinging on the total area of the holes. Finally, the transmitted light beam was collimated to a much higher degree than what was expected from the simple diffraction theory. Presumably, it was the periodic arrangement of holes that generated an interaction between the light beam and the metal surface across the total area of the metal film and holes that caused EOT. This experimental result inspired intense efforts to formulate a theoretical explanation. The picture that emerged was that the impinging light excited surface plasmons ${ }^{[20]}$ in the film surface. This excitation process was known from other experiments, as was the need of additional momentum since the photon and surface plasmon have different dispersion relations. In this case, the momentum difference, $\hbar q$ can be supplied (or absorbed) by the square lattice through the standard Umklapp relation:

$$
q=\frac{2 \pi}{a}
$$

where, $a$ is the lattice constant of the square lattice (i.e., the distance between the holes). The surface plasmons travel without loss across the metal surface and are transmitted as damped modes through the holes. Once at the exit surface, the surface plasmons interact, and are again emitted as photons, demonstrating it to be a well-collimated beam ${ }^{[21,22]}$. This explanation has been questioned $^{[23]}$ in the past but now appears to be accepted.

Two key conditions for the excitation apply to the two media, namely, air (I) and silver (II):

$$
\begin{aligned}
& \operatorname{Re}\left(\varepsilon_{\mathrm{II}}\right)<0 \\
& \left|\operatorname{Re}\left(\varepsilon_{\mathrm{II}}\right)\right|>\operatorname{Re}\left(\varepsilon_{\mathrm{I}}\right)>0 .
\end{aligned}
$$

At the excitation frequency, the real part of the silver film dielectric function must be negative and numerically larger than one. Furthermore, the film must be thin enough to limit the damping in the transmission process. These conditions are met in the infrared and part of the visible for thin films of high-conductivity metals. Later, it was noted that these requirements may also be satisfied in the Reststrahlen region of insulating compounds. Two recent reports on this option ${ }^{[24,25]}$ deal with the excitation of surface polaritons in $\mathrm{SiC}$.

The subset of Reststrahlen compounds that have their band in the thermal wavelength region of $5-25 \mu \mathrm{m}$ tends to be hard with high melting points ${ }^{[1]}$. This is a concern for their use in modern optoelectronic applications when thin films are needed in integrated components. In general, reactive sputtering is the most common, although not universal, solution to make thin films of these high-temperature compounds. Currently, there are two known reports regarding $\mathrm{BeO}$ sputtering ${ }^{[26,27]}$. SiC has been reactively direct-current (DC) sputtered, but the resulting film is a mixture of $3 \mathrm{C}$ and amorphous $\mathrm{SiC}^{[28]}$. Meanwhile, AlN is piezoelectric and is used in surface and bulk (surface acoustic wace (SAW) and bulk acoustic wave $($ BAW $)$ ) acoustic filters for mobile telephones ${ }^{[29,30]}$. This material can be deposited with sufficient purity and perfection for high-precision litography of small components. A simpler deposition process, intended for largearea solar energy applications ${ }^{[31]}$, has also been tried.

In conclusion, we have tried to cover the existing and potential applications of the reststrahlen band that is typical for polar compounds. It is "broad" only in the sense that we have included applications that are not only based on the interval of high reflectance, but also on typical features around the reststrahlen band following the oscillator model. With a few parameters, this model successfully predicts not only the interval with high reflectance, but also a narrow, deep reflectance minimum on the high photon energy side of the band. Continuing toward higher energy, the model also predicts a transition to dielectric behavior with low dispersion. Naturally, higher energy excitations will eventually interrupt this smooth behavior. The width of this interval is critically dependent upon whether the next excitation energy is another phonon in the meV range or the electronic energy gap in the $\mathrm{eV}$ range.

The applications we have cited from the literature include the uses of both the interval of high reflectance for monochromatization and that of the narrow low reflectance for wide angle AR filters. Opportunities to use a reststrahlen compound to modify the thermal emit- 
tance at moderately high temperatures have also been described, as well as the possibility to improve the performance with a single dielectric top layer on the compound.

\section{References}

1. C-G. Ribbing and E. Wäckelgård, Thin Solid Films 206, 312 (1991).

2. C. Kittel, Introduction to Solid State Physics (J. Wiley, New York, 1996).

3. J. A. Dobrowolski, Handbook of Optics (McGraw-Hill, New York, 1978).

4. A. Mitsuishi, Y. Yamada, and H. Yoshinaga, J. Opt. Soc. Am. 52, 14 (1962).

5. A. F. Turner, L. Chang, and T. P. Martin, Appl. Opt. 4, 927 (1965).

6. J. A. Dobrowolski, D. Poitras, P. Ma, H. Vakil, and M. Acree, Appl. Opt. 41, 3075 (2002).

7. D. Poitras and J. A. Dobrowolski, Appl. Opt. 43, 1286 (2004).

8. C. G. Ribbing, Opt. Lett. 15, 882 (1990).

9. C. G. Ribbing, Radiative control of outdoor condensation, Annales, Ac. Regia Sc. Upsaliensis 30, 115 (1993-94).

10. B. L. Adamson, "Fordonsruta av glas" Swedish. Patent 7609860-7 (1976).

11. I. Hamberg, J. S. E. M. Svensson, T. S. Eriksson, C. G. Granqvist, P. Arrenius, and F. Norin, Appl. Opt. 26, 2131 (1987).

12. T. Chibuye, C. G. Ribbing, and E. Wäckelgård, Appl. Opt. 33, 5975 (1994).

13. C-G. Ribbing, Ö. Staaf, and S. K. Andersson, Opt. Eng. 34, 3314 (1995).

14. H. Högström, G. Forssell, and C. G. Ribbing, Opt. Eng. 44, 026001 (2005).

15. C-G. Ribbing and A. Rung, "Sätt att skapa ett material and låg emittans i ett eller två bestämda våglängdsområden" Swedish. patent 0104195-3 (2003).

16. S. K. Andersson and C-G. Ribbing, Phys. Rev. B 49, 11336 (1994).

17. P. W. Baumeister and M. E. Krisl, "Article with thin film coating having an enhance emissivity and reduced absorption of radianr energy" U. S. Patent 4783373 (1988).

18. T. W. Ebbesen, H. J. Lezec, H. Ghaemi, T. Thio, and P. A. Wolf, Nature 391, 667 (1998).

19. H. Bethe, Phys. Rev. 66, 163 (1944).

20. H. Raether, Surface Plasmons-on Smooth and Rough surfaces and on Gratings (Springer, Berlin, 1988).

21. L. Martin-Moreno, F. J. Garcia-Vidal, H. J. Lezec, K. M. Pellerin, T. Thio, J. B. Pendry, and T. W. Ebbesen, Phys. Rev. Lett. 86, 1114 (2001).

22. L. Martin-Moreno, F. J. Garcia-Vidal, H. J. Lezec, A. Degiron, and T. W. Ebbesen, Phys. Rev. Lett. 90, 167401 (2003)

23. Q. Cao and P. Lalanne, Phys. Rev. Lett. 88, 057403 (2002).

24. P. B. Catrysse and S. Fan, Phys. Rev. B 75, 075422 (2007).

25. H. Högström, S. Valizadeh, and C. G. Ribbing, Opt. Mater. 30, 328 (2007).

26. D. Schalch, A. Scharmann, and A. Weiss, Thin Solid Films 124, 351 (1985).

27. T. Takagi, K. Matsubara, and H. Takaoka, J. Appl. Phys. 51, 5419 (1980).

28. Y. M. Lei, Y. H. Yua, L. L. Cheng, C. X. Ren, S. C. Zou, S. P. Wong, D. H. Chen, and I. H. Wilson, Mater. Lett. 43, 215 (2000).

29. R. Ruby, in Proceedings of 2007 IEEE International Ultrasonics Symposium 1029 (2007).

30. J. Kaitila, in Proceedings of 2007 IEEE International Ultrasonics Symposium 120 (2007).

31. S. Zhao and E. Wäckelgård, Sol. Energy Mater. Sol. Cell 90, 1861 (2006). 